Article

\title{
Numerical Study of Photoacoustic Pressure for Cancer Therapy
}

\author{
Thomas Grosges *,† and Dominique Barchiesi ${ }^{\dagger}$ \\ Group for Automatic Mesh Generation and Advanced Methods (Gamma3 UTT-INRIA), \\ University of Technology of Troyes, 12 rue Marie Curie-CS 42060, Troyes CEDEX 10004, France; \\ dominique.barchiesi@utt.fr \\ * Correspondence: thomas.grosges@utt.fr; Tel.: +33-3-25718430 \\ t These authors contributed equally to this work.
}

Academic Editor: Kohji Masuda

Received: 12 October 2016; Accepted: 11 November 2016; Published: 15 November 2016

\begin{abstract}
A commonly used therapy for cancer is based on the necrosis of cells induced by heating through the illumination of nanoparticles embedded in cells. Recently, the photoacoustic pressure shock induced by the illumination pulse was proved and this points to another means of cell destruction. The purpose of this study is to propose a model of the photoacoustic pressure in cells. The numerical resolution of the problem requires the accurate computation of the electromagnetism, the temperature and the pressure around the nanostructures embedded in a cell. Here, the problem of the interaction between an electromagnetic excitation and a gold nanoparticle embedded in a cell domain is solved. The variations of the thermal and photoacoustic pressures are studied in order to analyze the pressure shock wave inducing the collapse of the cell's membrane in cancer therapy.
\end{abstract}

Keywords: numerical approximation and analysis; medical optics and biotechnology; simulations; photoacoustics; thermal imaging; photothermal effects; photoacoustic therapy

\section{Introduction}

In the last decades, the theory and the applications of photoacoustics have been developed, in particular for the photothermal measurement techniques [1,2]. The applications of photoacoustics include spectroscopy, monitoring deexcitation processes, probing physical properties of materials, tomography and applications in photothermal therapy [3-5]. Photothermal therapy and photoacoustic techniques are procedures using laser illumination of photoabsorbers. Such therapies are non-invasive when working in the near-infrared spectrum. Metallic nanoparticles embedded in the tumor site absorb the laser irradiation and transfer the thermal energy to the surrounding medium (i.e., cancer cells) $[6,7]$. Photoacoustics refers to the generation of acoustic waves by modulating an optical radiation. Photoacoustic imaging is applied to measure temperature by tracking the temperature-induced changes in the photoacoustic signal amplitude [8].

In this context, we propose a numerical model for the accurate computation of photoacoustic pressure around gold nanopatricle embedded in a cell and the analysis of the spatio-temporal evolution of the pressure. The numerical method, including a 3D version of the adaptive remeshing scheme with error estimator $[9,10]$, takes into account the local and global variations of the pressure ensuring the convergence of the solution to the physical solution. The organization of the paper is as follows: Section 2 describes the equations of the problem and the numerical method. In Section 3, the results of the simulations are presented and a discussion on the ability of the pressure to destroy a cell is acheived before concluding the paper. 


\section{Photoacoustic Model}

This section presents the physical model and the numerical method used to solve the photoacoustic problem. The photoacoustic or thermoacoustic process refers to the generation of acoustic waves by absorption of electromagnetic energy. Such an absorption of the radiation by a nanoparticle acts like a heat source that depends on the space $\mathbf{x}$ and time $t$ and is given by:

$$
Q(\mathbf{x}, t)=\frac{\omega}{2} \epsilon_{0} \operatorname{Im}\left(\epsilon_{r}(\mathbf{x})\right)|\mathbf{E}(\mathbf{x}, t)|^{2}, \quad \forall \mathbf{x} \in \Omega,
$$

where $\omega$ is the angular frequency of the incoming wave, $\mathbf{E}$ the electric field, $\epsilon_{0}$ is the permittivity of vacuum, $\operatorname{Im}\left(\epsilon_{r}\right)$ is the imaginary part of $\epsilon_{r}$ the relative complex permittivity of materials that are functions of the $3 \mathrm{D}$ spatial coordinates $\mathbf{x}$ in the domaine $\Omega$. The laser illumination pulse satisfies the electromagnetic equation:

$$
\nabla \times\left(\frac{1}{\epsilon_{r}(\mathbf{x})} \nabla \times \mathbf{E}(\mathbf{x}, t)\right)-\frac{1}{c^{2}} \frac{\partial^{2} \mathbf{E}(\mathbf{x}, t)}{\partial t^{2}}=\mathbf{0}, \quad \forall \mathbf{x} \in \Omega,
$$

where $(\nabla \times)$ is the curl operator and $c$ the velocity of light. Equation (2) is solved with a radiation boundary condition $[11,12]$ at the external border $\Gamma$ of the computationnal domain $\Omega$ corresponding to the free propagation of the scattered field and the incident laser pulse of amplitude:

$$
E_{0}=\left[\frac{2 c \mu_{0} E_{\text {laser }}}{\pi R_{\text {beam }}^{2} \tau_{\text {pulse }}}\right]^{1 / 2},
$$

where $\mu_{0}$ is the permeability of the vacuum, $E_{\text {laser }}$ is the laser energy, $R_{\text {beam }}$ is the radius of the laser beam and $\tau_{\text {pulse }}$ the duration of the laser pulse. From such a heat source, the temperature elevation is governed by the equation:

$$
\left[\rho(\mathbf{x}) C_{p}(\mathbf{x}) \frac{\partial}{\partial t}-\nabla \cdot(\kappa(\mathbf{x}) \nabla)\right] T(\mathbf{x}, t)=Q(\mathbf{x}, t), \quad \forall \mathbf{x} \in \Omega,
$$

where $(\nabla$.$) and \nabla$ are the divengence and the gradient operators, $\rho$ is the mass density of the material, $C_{p}$ is the specific heat capacity, $\kappa$ the thermal conductivity and $T$ is the temperature. These are functions of time and of the 3D spatial coordinates $x$. Therefore, the resolution of the thermal problem requires solving the heat equation (Equation (4)) with a source $Q$ produced by the incoming electromagnetic illumination (Equation (1)) with the boundary condition $T(\mathbf{x}, t)=T_{0}$ a the boundary of the computational domain (i.e., $\forall \mathbf{x} \in \Gamma$ ). Such a thermal expansion induces acoustic displacement and the equation for photoacoustic generation is derived [2]:

$$
\left[\frac{1}{\rho(\mathbf{x}) v^{2}(\mathbf{x})} \frac{\partial^{2}}{\partial t^{2}}-\nabla \cdot\left(\frac{1}{\rho(\mathbf{x})} \nabla\right)\right] P(\mathbf{x}, t)=\beta(\mathbf{x}) \frac{\partial^{2}}{\partial t^{2}} T(\mathbf{x}, t), \quad \forall \mathbf{x} \in \Omega,
$$

with $v$ the sound velocity in the medium, $\beta$ is the expansion coefficient and $P$ is the acoustic pressure with initial and boundary conditions:

$$
P(\mathbf{x}, t=0)=p_{0} \quad \text { and } \quad n \cdot\left(\frac{1}{\rho(\mathbf{x})} \nabla\right) P(\mathbf{x}, t)=0, \quad \forall \mathbf{x} \in \Gamma,
$$

with $p_{0}$ being the initial pressure and $n$ the outgoing normal vector. The resolution of the weak coupled photothermal problem gives the spatio-temporal distribution of the pressure in the computational domain.

Equations (1)-(5) are numerically solved with a 3D Finite Element Method (FEM) with adaptive remeshing scheme using an a priori error estimator based on the Hessian of the solution calculated at 
the previous step of remeshing. The Finite Element Method (FEM) is now well known and has been applied for many years in mechanics, thermodynamics, electromagnetics and in electrical engineering $[13,14]$ and consists of solving systems of partial differential equation with boundary conditions in open or closed domains [11]. The general problem is solved on a discrete mesh of the domain [11] and the unknown physical quantities (E, T, P) are computed at the nodes of the mesh by using a variational method. The control of the error on the solution is achieved through an extended and improved 3D method, including a process of iterative remeshing $[9,10,12]$. The accuracy of the computed solutions are closely related to the quality of the mesh $[15,16]$. The quality of the solution is improved by adapting the size of the mesh elements to the physical solution $[10,12,17]$ through the remeshing process with adaptive loops. If strong variation is induced in the unknown physical quantities of interest, the convergence of the solution to a stable solution requires such mesh adaptions $[9,10]$. At each step of the adaption process, the approximations of the solutions of the thermic and acoustic wave equations $T$ and $P$, the temperature and the pressure are calculated in the basis of the interpolation polynomials between nodes. The maximum deviation between the solution associated with the mesh and the exact solution at nodes is limited by using the interpolation error (which is based on an estimation of the discrete Hessian of the solution) $[18,19]$ and is obtained from OPTIFORM software (adaptive remeshing generating isotropic or anisotropic meshes) [9], to govern the remeshing of the domain (with minimum and maximum element sizes $h_{\min }=0.1 \mathrm{~nm}$, $h_{\max }=100 \mathrm{~nm}$, and a tolerance on the relative error on the computed unknown physical quantities $\delta=0.001)$. Therefore, the domain is entirely remeshed each time and a new mesh is constructed.

\section{Numerical Results and Discussion}

Here, we consider a 3D spherical gold nanoparticle of radius $R_{p}=50 \mathrm{~nm}$ embedded in a 3D spherical cell of radius $R_{c}=1.0 \mu \mathrm{m}$ illuminated by an electromagnetic wave at $\lambda=532 \mathrm{~nm}$ and immersed in a water domain $\Omega$ of radius $R_{w}=22 \mu \mathrm{m}$ at initial temperature $T_{0}=37.5^{\circ} \mathrm{C}$ (i.e., $310.5 \mathrm{~K}$ ). This wavelength is chosen to be near the plasmon resonance for such a gold nanoparticle where light absorption is maximum and was already used as a contrast agent for in vivo tumor imaging [20]. The illumination pulse of duration $\tau_{\text {pulse }}=5 \mathrm{~ns}$ is achieved with a laser with energy $E_{\text {laser }}=10 \mathrm{~mJ}$ and a radius of the spotsize $R_{\text {beam }}=15 \mathrm{~mm}$.

The parameters of Equations (4)-(5) for the thermic and acoustic problems are given in Table 1.

Table 1. Values of the parameters for the photothermal model for the three media (water, cell, gold) and $j^{2}=-1$.

\begin{tabular}{|c|c|c|c|c|c|c|}
\hline Material & $\rho\left(\mathrm{kg} \cdot \mathrm{m}^{-3}\right)$ & $C_{p}\left(\mathrm{~m}^{2} \cdot \mathrm{s}^{-1} \cdot \mathrm{K}^{-1}\right)$ & $\kappa\left(\mathrm{kg} \cdot \mathrm{m} \cdot \mathrm{s}^{-3} \cdot \mathrm{K}^{-1}\right)$ & $v\left(\mathrm{~m} \cdot \mathrm{s}^{-1}\right)$ & $\beta\left(\mathrm{K}^{-1}\right)$ & $\epsilon_{r}$ \\
\hline water & 1000 & 4185 & 0.60 & 1493 & 0.000215 & 1.768 \\
\hline cell & 1090 & 2185 & 1.20 & 1535 & 0.000515 & 1.768 \\
\hline $\mathrm{Au}$ & 19,300 & 129 & 250 & 3240 & 0.000014 & $-5.58+\mathrm{j} 2.25$ \\
\hline
\end{tabular}

Figure 1a-d show the geometries of the problem with the meshes' associated materials for the computational domain and the cell, respectively. The relative permittivities of materials (which describe the electromagnetic problem of Equations (1) and (2)) are given in Table 1. Here, the materials of the problem are considered to be isotropic and homogeneous.

The results of the computation of the pressure maps are illustrated in Figure 2. Figure 2a-f shows the associated variations of the pressure at $0.5,1.5,2.5,4.5,14.5$ and $40 \mathrm{~ns}$ after the illumination. The variations and the propagation of the pressure wave is shown in the hole computational domain. The numerical scheme not only takes into account the shape and size of the nanoparticle but also the variations induced by the electromagnetism, the temperature and the pressure. The pressure is initiated at the beginning of the temperature shock that is induced by the modulation of the illumination. Such an acoustic pressure wave propagates in the whole media (cell and water, corresponding to a spatial extension of some micrometers the cell membrane has spatial extension from $-1 \mu \mathrm{m}$ to 
$1 \mu \mathrm{m}$ ) before vanishing. That contrasts with the temperature propagation (see Figure $3 \mathrm{a}, \mathrm{b}$ ) that is mainly located in the near vicinity of the gold nanoparticle (i.e., only a few nanometers from the gold nanoparticle, corresponding to the spatial expansion of the nanoparticle along the $y$-axis: -50 to $50 \mathrm{~nm}$ ). Therefore, only the nearest zone around the nanoparticle sees the temperature elevation. Figure 4 illustrates that the cell's membrane is receiving a pressure shock wave (i.e., the variation of pressure is non vanishing at the position of the cell membrane). Figure 5 shows the maximum of the pressure at the position of the cell membrane (i.e., at $1 \mu \mathrm{m}$ ) at the initiation of the temperature and pressure shock and the evolution of the pressure at the limit of the membrane. For an illumination at $\lambda=532 \mathrm{~nm}$, the two curves are obtained for two nanoparticle shapes. The first one consists of a spherical gold nanoparticle of radius $R=50 \mathrm{~nm}$, the second one is an ellipsoidal particle of semi-axis a $=100 \mathrm{~nm}$ and $b=50 \mathrm{~nm}$. Such a pressure variation can induce a deformation of the cell. The deformation is determined by the shear rate of the streaming flow field (computed from the strain tensor) and the cell elasticity [21,22]. Here, with the used materials, the shear stress components are: $\sigma_{z z}=\Delta P \approx 300 \mathrm{~Pa}$, $\sigma_{x x}=\sigma_{y y}=\Delta P R /(2 e) \approx 0.05 \mathrm{MPa}$ with $R$ being the radius of the cell, $e$ being the width of the cell's membrane and the associated shear modulus being $\tau \approx 0.01$ to $0.1 \mathrm{GPa}$ [23]. Moreover, the maximum rate of strain is about $S_{\max } \approx 10^{4}-10^{5} \mathrm{~s}^{-1}$ (i.e., $S_{\max } \approx \delta R / R / \Delta t$ [24] where $\Delta t$ is the evolution time). That leads to a maximum tension in the membrane $\tau_{\max } \approx \mu S_{\max } R$ varying in the range $\left[10^{-4} ; 10^{-1}\right] \mathrm{N} \cdot \mathrm{m}^{-1}$, where $\mu \approx 0.1$ to $0.001 \mathrm{~kg} \cdot \mathrm{m}^{-1} \cdot \mathrm{s}^{-1}$ is the dynamic viscosity of the aqueous solution in the cell (i.e., depending on the cell composition). Such a tension is sufficient to stabilize and neglect the thermal fluctuation of the membrane area, but will only expand the actual membrane area by a fraction $\Delta S / S \approx 2.0 \times 10^{-3}$ (with an expansion modulus of about $0.24 \mathrm{~N} \cdot \mathrm{m}^{-1}$ [24]). To achieve the shear of the membrane (or at least opening of pores), a ratio of area $\Delta S / S \geq 0.03$ is necessary [24]. To extend the application in cancer therapy, the illumination must use wavelengths in the biological window ( 800 to $1000 \mathrm{~nm}$ ). Such an illumination of a spherical gold nanoparticle at $\lambda=800 \mathrm{~nm}$ is shown in Figure 5. In such a window, the imaginary part of the permittivity of the gold nanoparticle is smaller and requires us to design the particle shape or to increase the energy laser [25]. Moreover, the use of larger cell (typically a cell size is varying from 1 to $100 \mu \mathrm{m}$ ) or a mix of larger nanoparticles, greater pulse energy or a higher repetition rate of ultra-short illumination shocks (which will induce an increase in the local pressure variation at the membrane boundary and therefore the maximum rate of strain) can open the way to a process inducing a local stress (shear stress) at the membrane in order to produce a disruption of the cell's membrane. Here, we focus on the study of one nanoparticle, nevertheless, metal nanoparticles can also be taken up by cells in 1-15 nanoparticles/vesicles [26,27]. The increase of the number of nanoparticles, if aggregated, has a similar effect of increasing the size and shape of the nanoparticle. Indeed, each nanoparticle plays the role of local temperature source inducing a photoacoustic pressure wave. Therefore, multiple pressure waves can be induced at the same time and the pressure shock at the membrane can also be increased due to the aggregated nanoparticles. Such a disruption could be produced by cytolysis (or osmotic lysis) that occurs when a cell bursts due to an osmotic imbalance that has caused excess water being released into the cell. 


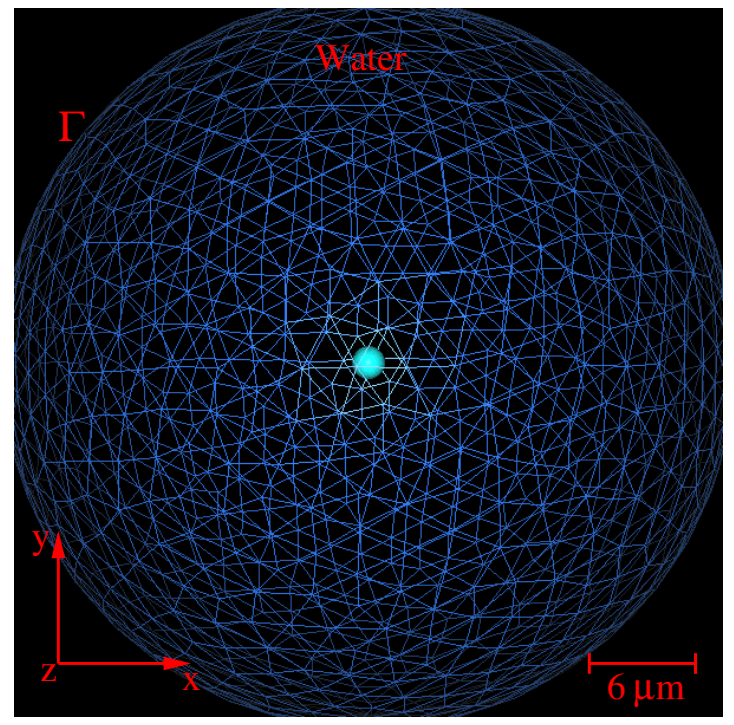

(a)

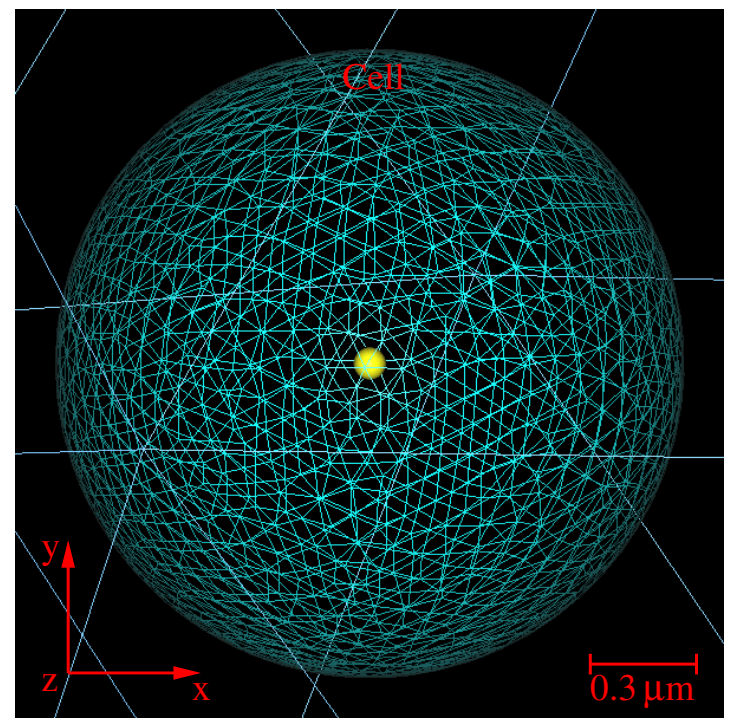

(c)

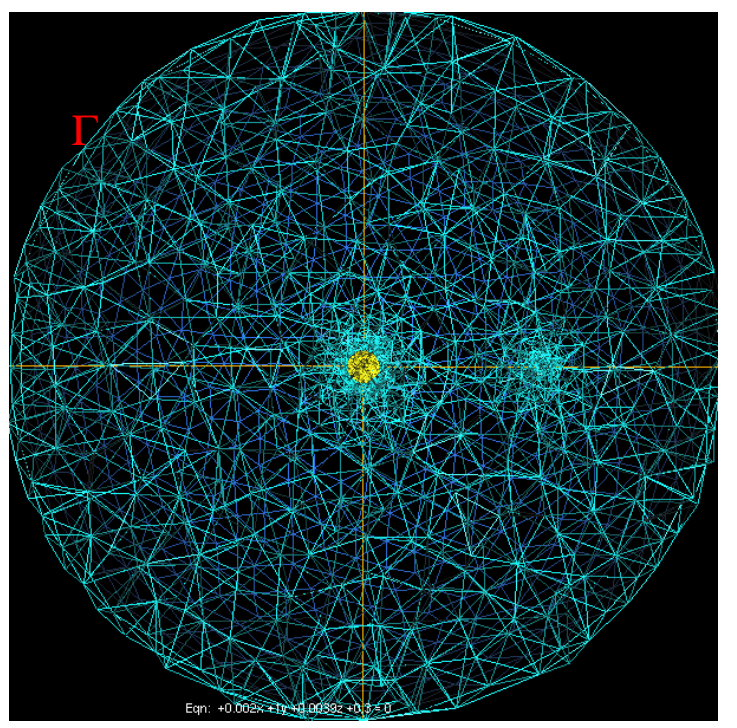

(b)

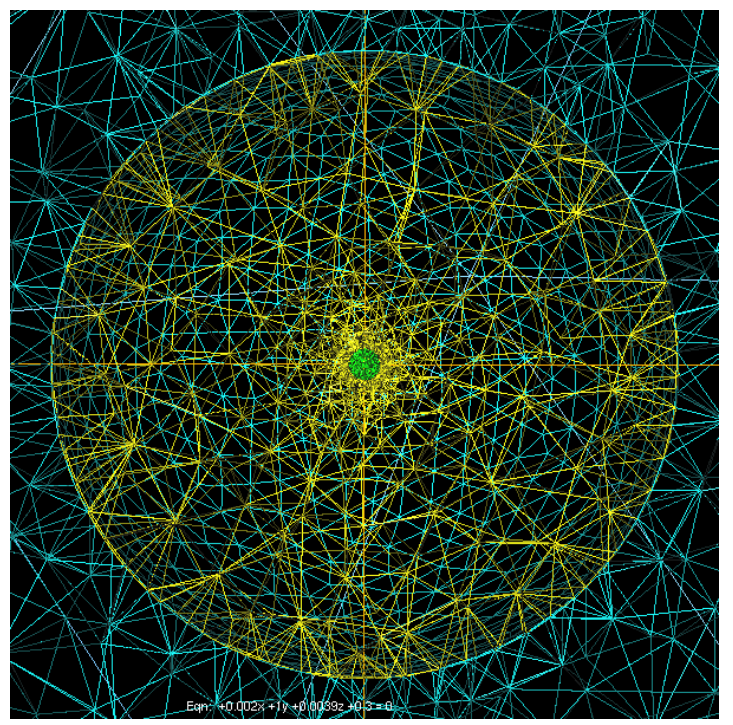

(d)

Figure 1. Geometry (a) and associated mesh (b) of the 3D water domain including the cell; Zoomed-in view in the xy plane on the cell (c) including the gold nanoparticle and (d) the associated mesh. 


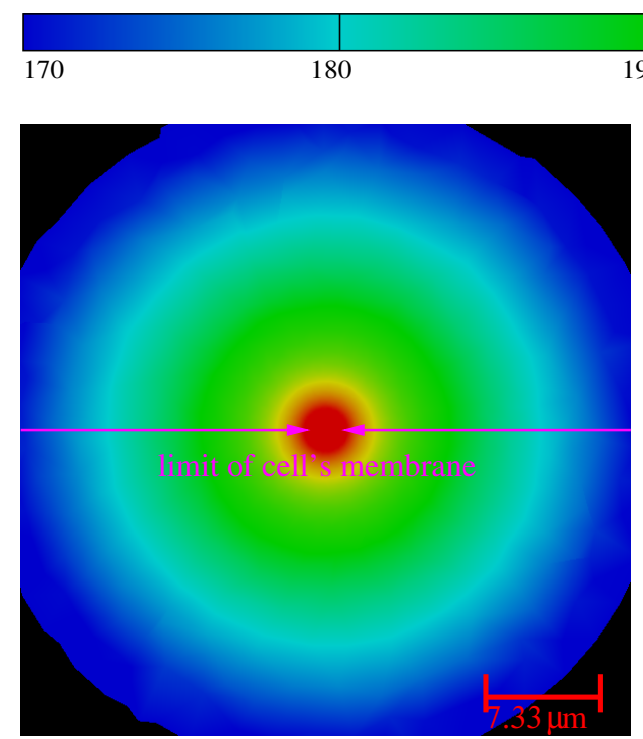

(a)



(c)

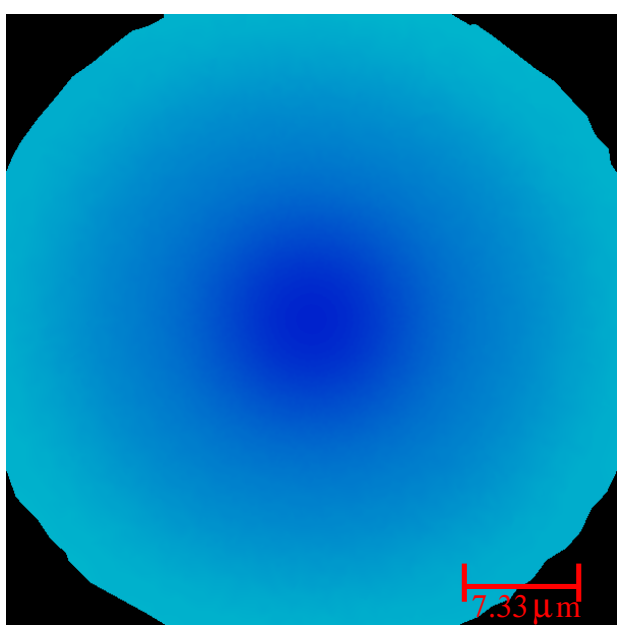

(e)

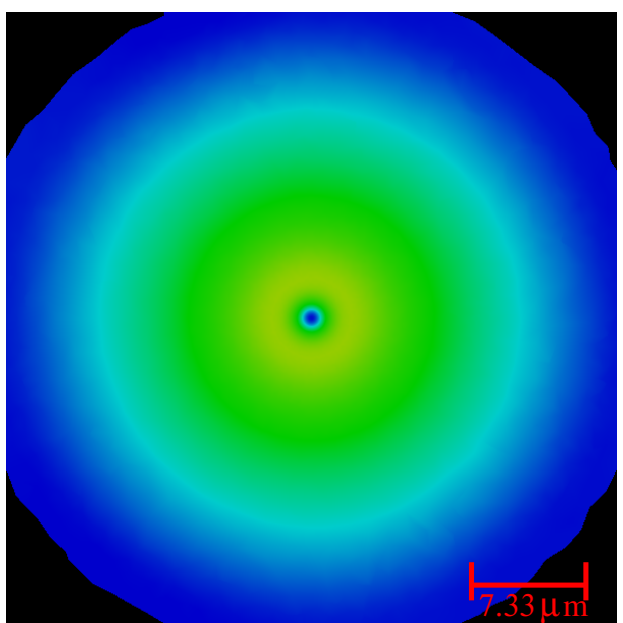

(b)



(d)



(f)

Figure 2. Time evolution of the variations of the acoustic pressure (in Pa) computed at (a) $0.5 \mathrm{~ns}$; (b) $1.5 \mathrm{~ns}$; (c) $2.5 \mathrm{~ns}$; (d) $4.5 \mathrm{~ns}$; (e) $14.5 \mathrm{~ns}$ and (f) $40.0 \mathrm{~ns}$ after the initial shock in the whole computationnal domain. The size of the cell's membrane is also illustrated at the beginning and the end of the wave propagation $(\mathbf{a}-\mathbf{f})$. The radii of the nanoparticle, the cell and the computationnal domain are: $50 \mathrm{~nm}, 1 \mu \mathrm{m}$ and $22 \mu \mathrm{m}$, respectively. 


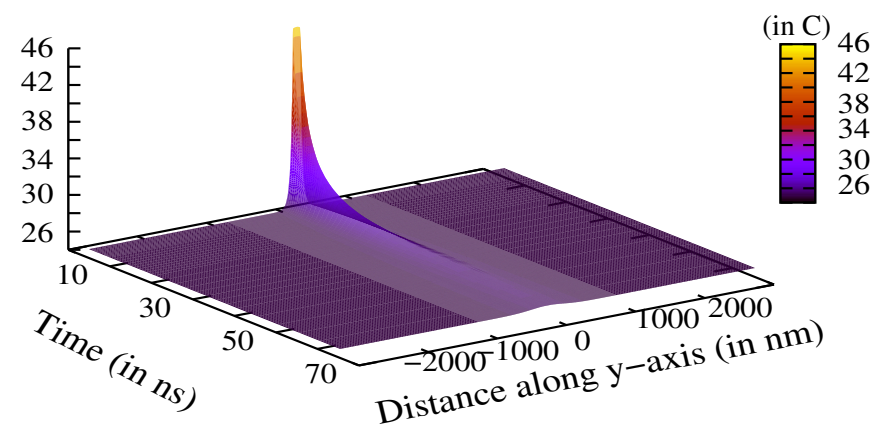

(a)

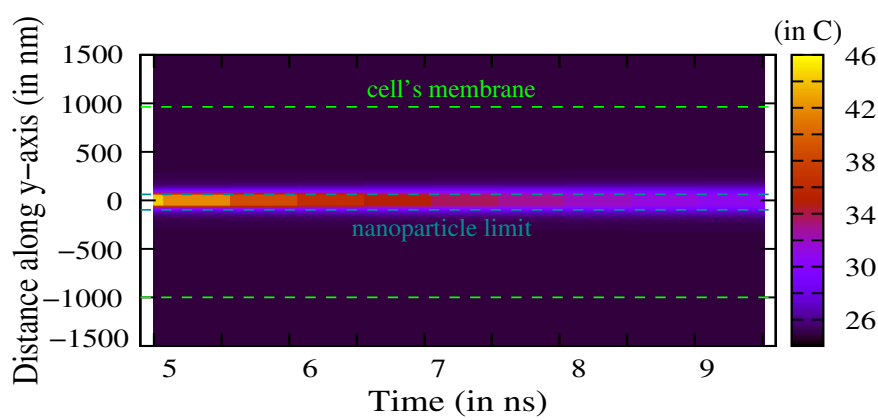

(b)

Figure 3. (a) Time evolution of the temperature (in ${ }^{\circ} \mathrm{C}$ ) in the cell (i.e., $|y| \leq 1000 \mathrm{~nm}$ ) and in the water (i.e., $|y| \geq 1000 \mathrm{~nm}$ ); (b) The spatial distribution of the temperature stays located in the near vicinity of the nanoparticle (i.e., at $|y| \approx 50 \mathrm{~nm}$ ).

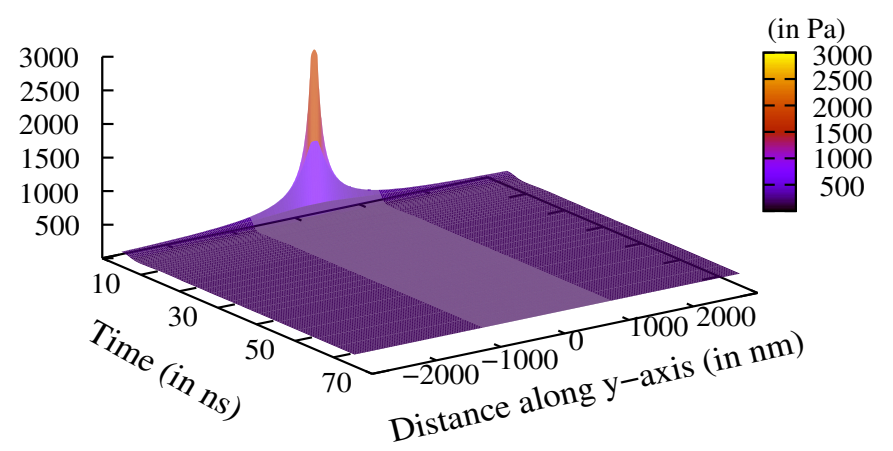

(a)

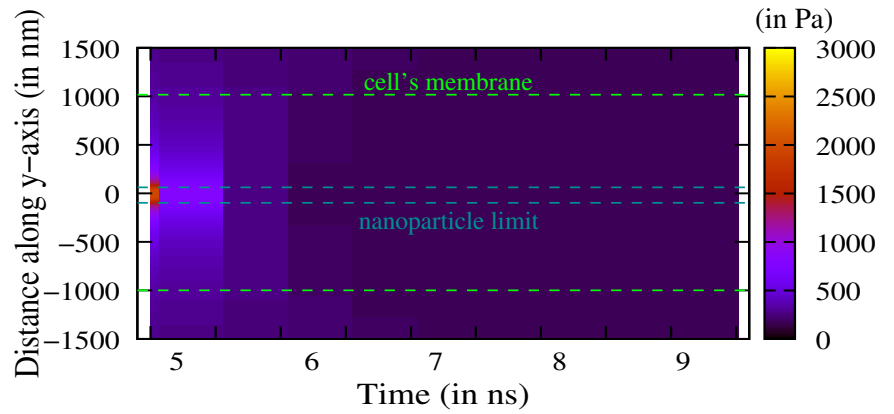

(b)

Figure 4. (a) Time evolution of the variation of the pressure (in Pa) in the cell (i.e., $|y| \leq 1000 \mathrm{~nm}$ ) and in the water (i.e., $|y| \geq 1000 \mathrm{~nm}$ ); (b) The spatial distribution of the pressure variation is computed from the center of the nanoparticle (i.e., at $|y| \approx 0 \mathrm{~nm}$ ) and propagated to the cell membrane (i.e., at $|y| \approx 1 \mu \mathrm{m}$ ). 




Figure 5. Evolution of the variation of pressure at the limit of the cell membrane as a function of time, along the $y$-axis for two nanoparticle shapes (spheroide of radius $\mathrm{R}=50 \mathrm{~nm}$ illuminated at $\lambda=532 \mathrm{~nm}$ and $\lambda=800 \mathrm{~nm}$, and an ellipsoide of semi-axis $\mathrm{a}=100 \mathrm{~nm}, \mathrm{~b}=50 \mathrm{~nm}$ illuminated at $\lambda=532 \mathrm{~nm}$ ).

\section{Conclusions}

The paper focuses on the analysis of the direct 3D numerical computation of the time-dependant photoacoustic pressure of a gold nanoparticle embedded in a cell. The adaption of the computation process was achieved to optimize both the temperature amplitude and the propagation of the acoustic wave pressure. The variation of the pressure at the limit of the cell's membrane relative to the time of excitation is presented. We discuss the ability of the use of pressure to disrupt the cell membrane, which can be an alternative way to induce lysis of the membrane. The use of complex particles or cell shapes can extend the domain of its application, especially to the study and the design of a pressure-based non invasive theragnostic for cancer.

Acknowledgments: This research did not receive any specific grant from funding agencies in the public, commercial, or not-for-profit sectors.

Author Contributions: Thomas Grosges conceived and performed the modelling; Thomas Grosges, and Dominique Barchiesi analyzed the results; Dominique Barchiesi contributed reagents/analysis/modelling tools; Thomas Grosges wrote the paper.

Conflicts of Interest: The authors declare no conflict of interest.

\section{References}

1. Shah, J.; Park, S.; Aglyamov, S.; Larson, T.; Ma, L.; Sokolov, K.; Johnston, K.; Milner, T.; Emelianov, S.Y. Photoacoustic imaging and temperature measurement for photothermal cancer therapy. J. Biomed. Opt. 2008, 13, 034024 .

2. Tam, A.C. Applications of photoacoustinc sensig techniques. Rev. Mod. Phys. 1986, 58, 381-431.

3. Kruger, A.; Fang, P.; Appledorn, Y.R. Photoacoustic ultrasound (paus)-reconstruction tomography. Med. Phys. 1995, 22, 1605-1609.

4. Wang, X.; Xu, Y.; Xu, M.; Yokoo, S.; Fry, E.S.; Wang, L.V. Photoacoustic tomography of biological tissues with high cross-section resolution: Reconstruction and experiment. Med. Phys. 2002, 29, 2799-2805.

5. Oraevsky, A.A.; Karabutoc, A.A. Optoacoustic Tomography; CRC Press: Boca Raton, FL, USA, 2003.

6. Huang, X.; El-Sayed, I.H.; Qian, W.; El-Sayed, M.A. Cancer cell imaging and photothermal therapy in the near-infrared region by using gold nanorods. J. Am. Chem. Soc. 2006, 128, 2115-2120.

7. Loo, C.; Lin, A.; Hirsch, L.; Lee, M.H.; Barton, J.; Halas, N.; West, J.; Drezek, R. Nanoshell-enabled photonics-based imaging and therapy of cancer. Technol. Cancer Res. Treat. 2004, 3, 33-40.

8. Larimam, I.V.; Larin, K.V.; Esenaliev, R.O. Real-time optoacoustic monitoring of temperature in tissues. J. Phys. D 2005, 38, 2633-2639. 
9. Borouchaki, H.; Grosges, T.; Barchiesi, D. Improved 3D adaptive remeshing scheme applied in high electromagnetic field gradient computation. Finite Elem. Anal. Des. 2010, 46, 84-95.

10. Grosges, T.; Borouchaki, H.; Barchiesi, D. Three dimensional adaptive meshing scheme applied to the control of the spatial representation of complex field pattern in electromagnetics. Appl. Phys. B Lasers Opt. 2010, 101, 883-889.

11. Jin, J. The Finite Element Method in Electromagnetics; John Wiley and Sons: New York, NY, USA, 1993.

12. Mezghani, F.; Barchiesi, D.; Cherouat, A.; Grosges, T.; Borouchaki, H. Comparison of 3D Adaptive Remeshing Strategies for Finite Element Simulations of Electromagnetic Heating of Gold Nanoparticles. Adv. Math. Phys. 2015, 2015, 469310.

13. Courant, R. Variational methods for the solution of problems of equilibrium and vibrations. Bull. Am. Math. Soc. 1943, 49, 1-23.

14. Silvester, P.; Pelosi, G. Finite Elements for Wave Electromagnetics: Methods and Techniques; IEEE Press: New York, NY, USA, 1994.

15. Ciarlet, P.G. Basic Error Estimates for Elliptic Problems; North-Holland: Amsterdam, The Netherlands, 1991.

16. Xue, D.; Demkowicz, L. Modeling of electromagnetic absorption/scattering problems on curvilinear geometries using hp finite/infinite element method. Finite Elem. Anal. Des. 2006, 42, 570-579.

17. Bourouchaki, H.; Grosges, T.; Barchiesi, D. Enhancement of the accuracy of numerical field computation using adaptive three dimensional remeshing scheme. C. R. Mec. 2010, 338, 127-131.

18. Radovitzky, R.; Ortiz, M. Error estimation and adaptive meshing in strongly non-linear dynamic problems. Comput. Method Appl. Mech. Eng. 1999, 172, 203-240.

19. Ainsworth, M.; Oden, J.T. A posteriori error estimation in finite element analysis. Comput. Method Appl. Mech. Eng. 1997, 142, 1-88.

20. Zhang, Q.; Iwakuma, N.; Shaarma, P.; Moudgil, B.M.; Wu, C.; Mc Neill, J.; Jiang, H.; Grobmeyer, S.R. Gold nanoparticles as a contrast aggent for in vivo tumor imaging with photoacoustic tomography. Nanotechnol 2009, 20, 395102.

21. Marmottant, P.; Hilgenfeldt, S. Controlled vesicle deformation and lysis by single oscillating bubbles. Nature 2003, 423, 153-156.

22. Boal, D. Mechanics of the Cell; Cambridge University Press: Cambridge, UK, 2002.

23. Hibbeler, R.C. Mechanics of Materials; Pearson Prentice Hall: Upper Saddle River, NJ, USA, 2004.

24. Seifert, U. Fluid membranes in hydrodynamic flow fields: Formalism and an application to fluctuating quasispherical vesicles in shear flow. Eur. Phys. J. B 1999, 8, 405-415.

25. Grosges, T.; Barchiesi, D.; Kessentini, S.; Gréhan, G.; Lamy de la Chapelle, M. Nanoshells for photothermal therapy: A Monte-Carlo based numerical study of their design tolerance. Biomed. Opt. Express 2011, 2, 1584-1596.

26. Fan, L.L.; Zhu, M.; Lee, X.; Zhang, R.J.; Wang, K.L.; Wei, J.Q.; Zhong, M.L.; Wu, D.H.; Zhu, H.W. Direct Synthesis of Graphene Quantum Dots by Chemical Vapor Deposit. Part. Part. Syst. Charact. 2013, 30, 764-769.

27. Bergamini, L.; Voliani, V.; Cappello, V.; Nifosi, R.; Corni, S. Non-linear optical response by functionalized gold nanospheres: Identifying design principles to maximize the molecular photo-release. Nanoscale 2015, 7, 13345-13357.

(C) 2016 by the authors; licensee MDPI, Basel, Switzerland. This article is an open access article distributed under the terms and conditions of the Creative Commons Attribution (CC-BY) license (http://creativecommons.org/licenses/by/4.0/). 